\title{
Review Article \\ Plasma Formation during Acoustic Cavitation: Toward a New Paradigm for Sonochemistry
}

\author{
Sergey I. Nikitenko \\ Institut de Chimie Séparative de Marcoule, UMR 5257-CEA-CNRS-UMII-ENSCM, Centre de Marcoule, Bâtiment 426, BP 17171, 30207 \\ Bagnols-sur-Cèze Cedex, France
}

Correspondence should be addressed to Sergey I. Nikitenko; serguei.nikitenko@cea.fr

Received 12 February 2014; Accepted 9 April 2014; Published 4 May 2014

Academic Editor: Samir K. Pal

Copyright (C) 2014 Sergey I. Nikitenko. This is an open access article distributed under the Creative Commons Attribution License, which permits unrestricted use, distribution, and reproduction in any medium, provided the original work is properly cited.

The most recent spectroscopic studies of single bubble (SBSL) and multibubble (MBSL) sonoluminescence reveal that the origin of extreme intrabubble conditions is related to nonequilibrium plasma formed inside the collapsing bubbles. Analysis of the relative populations of $\mathrm{OH}\left(\mathrm{A}^{2} \Sigma^{+}\right)$vibrational states observed during MBSL in water saturated with noble gases shows that in the presence of argon at low ultrasonic frequency weakly excited plasma is formed. At high-frequency ultrasound the plasma inside the collapsing bubbles exhibits Treanor behavior typical for strong vibrational excitation. Plasma formation during SBSL was observed in concentrated $\mathrm{H}_{2} \mathrm{SO}_{4}$ preequilibrated with Ar. The light emission spectra exhibit the lines from excited Ar atoms and ionized oxygen $\mathrm{O}_{2}{ }^{+}$. Formation of $\mathrm{O}_{2}{ }^{+}$species is inconsistent with any thermal process. Furthermore, the SBSL spectra in $\mathrm{H}_{2} \mathrm{SO}_{4}$ show emission lines from $\mathrm{Xe}^{+}, \mathrm{Kr}^{+}$, and $\mathrm{Ar}^{+}$in full agreement with plasma hypothesis. The photons and the "hot" particles generated by cavitation bubbles enable the excitation of nonvolatile species in solutions increasing their chemical reactivity. Secondary sonochemical products may arise from chemically active species that are formed inside the bubble but then diffuse into the liquid phase and react with solution precursors to form a variety of products.

\section{Introduction}

When studying the action of $100-500 \mathrm{kHz}$ ultrasound on aqueous solutions, Richards and Loomis [1] discovered that the ultrasonic waves accelerate the hydrolysis of dimethylsulfate and the reduction of potassium iodate by sulfurous acid (iodine "clock" reaction). Two years later, Schmitt et al. [2] reported the oxidation of iodide ions in aqueous solutions under the effect of $750 \mathrm{kHz}$ ultrasound. These seminal works have introduced a new field of chemistry called "sonochemistry" by Neppiras [3]. Large amounts of research papers and detailed critical reviews have been published since that time describing different ultrasonic processes, such as cleaning and degassing [4], extraction of biologically active compounds [5], food processing [6,7], advanced oxidation processes $[8,9]$, synthesis of nanostructured materials [10], and redox reactions of actinide ions [11].

Ultrasound spans the frequencies of roughly $15 \mathrm{kHz}$ to $1 \mathrm{GHz}$. As a rule, the sonochemical effects are observed in the range of $15 \mathrm{kHz}-2 \mathrm{MHz}$. The ultrasound of higher frequency is used for medical and material diagnostics [4]. The acoustic wavelengths of chemically active ultrasound (1$10^{-4} \mathrm{~cm}$ ) are much higher than the molecule size. Therefore, the sonochemistry arises not from a direct action of ultrasonic waves on molecules, but rather from the acoustic cavitation. Simply put, cavitation is a set of consequent events: nucleation, growth, and violent collapse of microbubbles in liquids submitted to ultrasonic vibrations. There is a general consensus that the chemical and physical effects of power ultrasound are related to extremely rapid implosion of the cavitation bubbles occurring at the final stage of collapse.

It is important to emphasize that the acoustic cavitation leads not only to the chemical transformation of the medium, but also to the light emission, known as sonoluminescence (SL). In 1933 Marinesco and Trillat have accidentally observed the darkening of photographic plates submitted to ultrasound in water [12]. They attributed this finding to the ultrasonic acceleration of $\mathrm{Ag}^{+}$chemical reduction at the surface of plates. However, one year later, Frenzel and Schultes [13] have shown that photographic plate darkening is due to 
the light emission from sonicated water rather than from chemical reaction. In modern literature, two different types of SL can be distinguished: light emission from a cloud of bubbles (multibubble sonoluminescence (MBSL) and light emission from a single cavitation bubble trapped in a standing acoustic wave of relatively weak acoustic pressure (single bubble sonoluminescence (SBSL). For the first time SBSL was reported by Yosioka and Omura [14]. Eight years later, Temple described the same phenomenon in his MS thesis [15]. However, both observations did not attract the attention of scientific community and only in 1990 Gaitan and Crum independently rediscovered and studied more in detail the SBSL in water/glycerin mixtures [16]. In degassed water, the SBSL was bright enough to be visible to the naked eye. The experiments demonstrated the unique properties of this system: the light emission occurs at every acoustic cycle at the final stage of collapse and the pulse duration of the light flash is below 200 ps [17-19]. Note here that single collapsing bubbles can be produced in liquids also by focused laser beam [20] but the sonoluminescence of such kind of bubbles is less studied than that of ultrasonic bubbles. In the years since SBSL was discovered, more than thousand papers have been published on this topic. The most significant results in sonoluminescence obtained before 2005 have been summarized in the monograph of Young [21]. This review will be focused on more recent results in sonoluminescence and sonochemistry which provided the new insights into the origin of the processes occurring during acoustic cavitation.

\section{Sonoluminescence as a Probe of Intrabubble Conditions}

The nonlinear oscillations of a bubble driven in liquids by acoustic waves are generally described by Rayleigh-Plesset equation [23-26]:

$$
R \ddot{R}+\frac{3}{2} \dot{R}^{2}=\frac{1}{\rho}\left[\left(P_{0}+\frac{2 \sigma}{R_{0}}\right)\left(\frac{R_{0}}{R}\right)^{3 \gamma}-\frac{2 \sigma}{R}-\frac{4 \mu \dot{R}}{R}+P_{\infty}\right],
$$

where $R$ is the bubble radius, $\dot{R}$ and $\ddot{R}$ are the bubble wall velocity and the acceleration, respectively, $R_{0}$ is the equilibrium bubble radius at ambient pressure $P_{0}, \gamma$ is the polytropic index, $\rho$ is the bulk density, $\sigma$ is the surface tension, $\mu$ is the viscosity, and $P_{\infty}$ is the far-field acoustic pressure. Numerous studies of bubble dynamics reviewed recently by Lauterborn and Kurz indicate that the RayleighPlesset equation works well over most of the range of bubble radius [27]. However, this equation fails at the final stage of bubble implosion where the density of compressed gas inside the bubble is comparable with that of the bulk liquid [28]. It is noteworthy that the SL flash occurs only in the last stage of collapse. Consequently, the theoretical description of conditions required for SL based on Rayleigh-Plesset equation becomes somewhat uncertain. Despite this limitation, (1) is very useful for calculations of several practically important parameters of cavitation bubbles. For example, Table 1 summarizes the calculated values of maximal bubble radius $\left(R_{m}\right)$ and the time of total collapse $(\tau)$ as a function
TABLE 1: The values of maximal bubble radius $\left(R_{m}\right)$ and its collapse time $(\tau)$ as a function of ultrasonic frequency $(f)$ in air-saturated water and at ultrasonic intensity of $10 \mathrm{~W} \cdot \mathrm{cm}^{-2}$.

\begin{tabular}{lcc}
\hline$f, \mathrm{kHz}$ & $R_{m}, \mu \mathrm{m}$ & $\tau, \mu \mathrm{s}$ \\
\hline 20 & 150 & 5 \\
205 & 17.5 & 1.6 \\
358 & 10.0 & 0.9 \\
618 & 5.8 & 0.5 \\
1071 & 3.3 & 0.3 \\
\hline
\end{tabular}

of ultrasonic frequency presuming Minnaert's acoustic resonance conditions [29]. One can see that the bubbles are much smaller and their implosion is much more rapid at higher ultrasonic frequency. It should be emphasized that the distribution of the cavitation field is also different between low- and high-frequency ultrasound. A $20 \mathrm{kHz}$ horn system yields a limited conical cavitation zone near the horn tip while high frequencies $(\geq 100 \mathrm{kHz})$ tend to give a more diffuse, widely distributed zone of cavitation [4]. Therefore, at high frequency, a larger volume of solution is directly submitted to ultrasonic irradiation compared to $20 \mathrm{kHz}$ ultrasound. On the other hand, as the frequency increases, the generation of cavitation bubbles becomes more difficult to achieve in the available time and that greater sound intensity is needed to provide bubble formation.

In the pioneering work on acoustic cavitation, Rayleigh suggested that the rapid bubble implosion should lead to adiabatic heating of the gas inside the cavity [30]. This idea was further developed by Noltingk and Neppiras [31] and later by Flynn [32]. Using Rayleigh-Plesset equation and presuming adiabatic conditions during bubble implosion, they calculated the temperature and the pressure within the bubble at the moment of total collapse. For nitrogenfilled bubble in water, there calculations provided $4200 \mathrm{~K}$ and 975 atm, respectively. Note here that this calculation does not take into account electronic and vibrational excitation of $\mathrm{N}_{2}$ molecules or their possible high temperature dissociation. Despite the numerous contradicting points noted by Margulis [33], the adiabatic heating model based on simple physical principles was quite rapidly accepted by sonochemists since it allowed interpreting the most of sonochemical phenomena in terms of easily understandable flame-like processes.

Spectroscopic studies of sonoluminescence offer a powerful experimental tool to probe the intrabubble conditions and to confirm or to improve the theoretical models of cavitation. The SBSL spectra of degassed water did not reveal any structures as lines and bands. Early SBSL studied attributed these spectra to blackbody emission which is in line with adiabatic heating model. However, Gompf at al. [34], Hiller et al. [35], and Moran and Sweider [36] have not found any significant difference between the pulse width in the UV part of SBSL spectrum (300-400 nm) and in the red part (590-650 nm). This contradicts a thermal model which suggests that the red pulse of the black body emission should be about twice as long as the UV part. Moreover, the intensity of the pulse predicted by blackbody model is 
about two orders of magnitude larger than the experimental values for studied experimental parameters [37]. Today many researches believe that the featureless SBSL spectra originated from bremsstrahlung rather than from blackbody emission $[21,37]$. Bremsstrahlung emission is produced by the deceleration of an electron by an atomic nucleus in plasma. Plasma formation in single cavitation bubble was observed in concentrated $\mathrm{H}_{2} \mathrm{SO}_{4}$ preequilibrated with 50 mbar of argon at the driven frequency of $20 \mathrm{kHz}$ [38]. Spectral analysis revealed light emission from excited Ar atoms and ionized oxygen $\mathrm{O}_{2}{ }^{+}$. Formation of $\mathrm{O}_{2}{ }^{+}$species is inconsistent with any thermal process. The ionization energy of $\mathrm{O}_{2}$ molecules is more than twice its bond dissociation energy. Therefore, the strong heat should lead to $\mathrm{O}_{2}$ homolytic dissociation rather than to ionization. By contrast, the $\mathrm{O}_{2}{ }^{+}$species can be formed by electron impact in plasma [39]. Furthermore, the SBSL spectra in sulfuric acid in the presence of noble gases show emission line from $\mathrm{Xe}^{+}, \mathrm{Kr}^{+}$, and $\mathrm{Ar}^{+}$with the energies ranging from $26.0 \mathrm{eV}$ to $34.2 \mathrm{eV}$ [40]. On the other hand, the effective gas temperature calculated from $\mathrm{Ar}^{*}$ line widths at the same conditions is only about $1 \mathrm{eV}$ (ca. $11000 \mathrm{~K}$ ). Such a discrepancy cannot be understood by presuming only adiabatic heating during bubble collapse. The time-resolved spectra of SBSL in $\mathrm{H}_{2} \mathrm{SO}_{4}$ preequilibrated with $\mathrm{Kr}$ measured using streak camera revealed the spectrum evolution with time of collapse [41]. At $0.5 \mathrm{~ns}$ the SBSL spectrum exhibits line emission from excited $\mathrm{Kr}$ atoms centered at $810 \mathrm{~nm}$ (5s-5p). With evolution of time, the lines of $\mathrm{Kr}^{*}$ disappear, the central wavelength moves from infrared to ultraviolet monotonously, and at $8.5 \mathrm{~ns}$ the spectrum shows featureless emission in the UV range. Emission from excited $\mathrm{Kr}(E \sim 9.9 \mathrm{eV})$ at the early stage of collapse is incompatible with the adiabatic heating model as a possible mechanism of SBSL.

In contrast to SBSL, the MBSL spectra in different solvents exhibit molecular lines emission in a wide range of conditions. Spectroscopic analysis of $\mathrm{C}_{2}{ }^{*}$ emission bands (Swan band $\mathrm{d}^{3} \Pi_{\mathrm{g}}-\mathrm{a}^{3} \Pi_{\mathrm{u}}$ ) during sonolysis at $20 \mathrm{kHz}$ of argonsaturated silicon oil gives the effective cavitation temperature of about $5000 \mathrm{~K}$ [42]. However, the interpretation of this spectroscopic temperature is not without complications since $\mathrm{C}_{2}{ }^{*}$ is the product of a set of chemical reactions, and the relative band intensities might reflect the kinetics of these reactions rather than the intrabubble temperature. The MBSL spectra of $\mathrm{H}_{2} \mathrm{SO}_{4}$ at $20 \mathrm{kHz}$ in the presence of argon revealed the emission lines from excited $\mathrm{Ar}^{*}$ atoms [43]. However, like in the case of SBSL, the calculated effective gas temperature $(\sim 8000 \mathrm{~K})$ was much lower than the energy of $\mathrm{Ar}^{*}$ species ( $13 \mathrm{eV} \sim 150900 \mathrm{~K})$.

The MBSL spectra of pure water preequilibrated with $\mathrm{Ar}, \mathrm{Kr}$, and $\mathrm{Xe}$ are composed of the emission lines of excited $\mathrm{OH}^{*}$ radicals in $\mathrm{A}^{2} \Sigma^{+}$and $\mathrm{C}^{2} \Sigma^{+}$states and a broad continuum ranging from UV to near-infrared spectral range, which probably results from the superposition of several emission bands: $\mathrm{H}+\mathrm{OH}^{*}$ recombination, water molecule de-excitation, and $\mathrm{OH}\left(\mathrm{B}^{2} \Sigma^{+}-\mathrm{A}^{2} \Sigma\right)$ emission $[22,44]$. Figure 1 shows the experimental SL spectra in argon measured at different ultrasonic frequencies normalized on the most intense $\mathrm{OH}\left(\mathrm{A}^{2} \Sigma^{+}-\mathrm{X}^{2} \Pi_{\mathrm{i}}\right)(0-0)$ transition. In the spectral

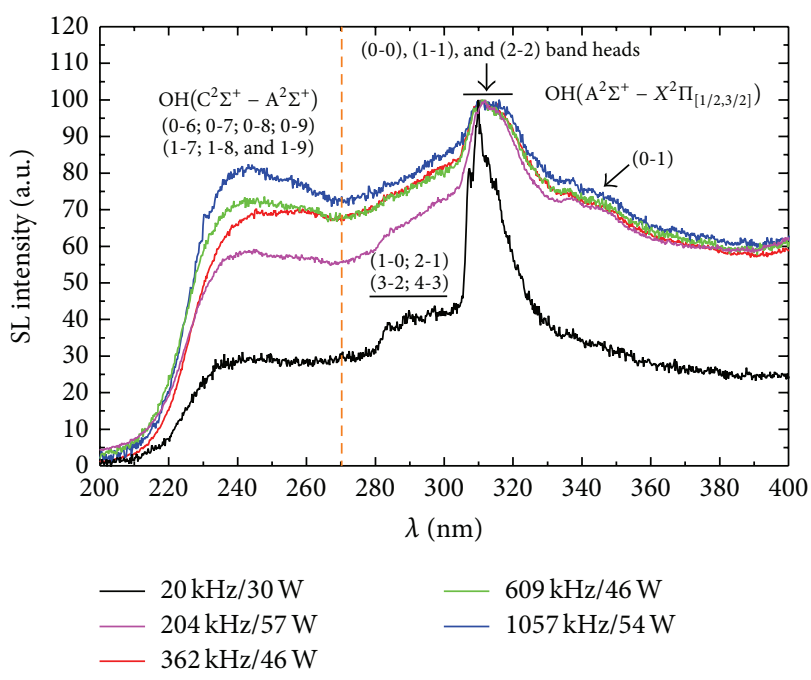

FIGURE 1: Normalized MBSL spectra of water sparged with argon at $10-11^{\circ} \mathrm{C}$ for different ultrasonic frequencies. Reproduced with permission from [22].

range of $280-350 \mathrm{~nm}$, the vibrational bands (0-0), (0-1), (1-0), (1-1), (2-1), (2-2), (3-2), and (4-3) of the $\mathrm{OH}(\mathrm{A}-\mathrm{X})$ system can be identified. These spectra clearly demonstrate the dramatic effect of ultrasonic frequency on the relative populations of vibrational levels. In addition, for high-frequency ultrasound, $\mathrm{OH}\left(\mathrm{C}^{2} \Sigma^{+}-\mathrm{A}^{2} \Sigma^{+}\right)$emission can be seen around $250 \mathrm{~nm}$. The $\mathrm{OH}\left(\mathrm{C}^{2} \Sigma^{+}\right)$state cannot be populated by any thermal process since excited water molecule required for formation of such species must possess an excitation energy of at least $16.1 \mathrm{eV}$. The most probable mechanism of $\mathrm{OH}\left(\mathrm{C}^{2} \Sigma^{+}\right)$species production involves electron impact of water molecules. It was concluded that the observation of $\mathrm{OH}\left(\mathrm{C}^{2} \Sigma^{+}-\mathrm{A}^{2} \Sigma^{+}\right)$ emission indicates the formation of nonthermal plasma inside the cavitation bubble [44]. Spectroscopic analysis of $\mathrm{OH}\left(\mathrm{A}^{2} \Sigma^{+}-\mathrm{X}^{2} \Pi_{\mathrm{i}}\right)$ vibrational transitions supports this hypothesis [22]. The relative populations of $\mathrm{OH}\left(\mathrm{A}^{2} \Sigma^{+}\right)$ $v^{\prime}=1-4$ vibrational states have been calculated using an optical thin plasma model. Figure 2 shows that, in contrast to a thermalized system, the relative population distribution obtained from MBSL spectra deviates strongly from the equilibrium Boltzmann distribution. At $20 \mathrm{kHz}$, the vibrational population distribution of $\mathrm{OH}\left(\mathrm{A}^{2} \Sigma^{+}\right)$state appears to follow a Brau distribution function typical for weak vibrational excitation [39]. At higher ultrasonic frequencies, it follows a Treanor distribution function which describes strong vibrational excitation [39]. It is noteworthy that the spectroscopic analysis based on the determination of the relative vibrational level populations of excited species does not depend on total intensity of sonoluminescence. Consequently, this method is sensitive to intrabubble conditions rather than to the total number of light emitting bubbles. Obviously, plasma far from equilibrium cannot be characterized by average gas temperature around $5000 \mathrm{~K}$ usually referred to multibubble cavitation in water [28]. Such kind of plasma is described by multiple temperatures related to different plasma particles and different degrees of freedom. 


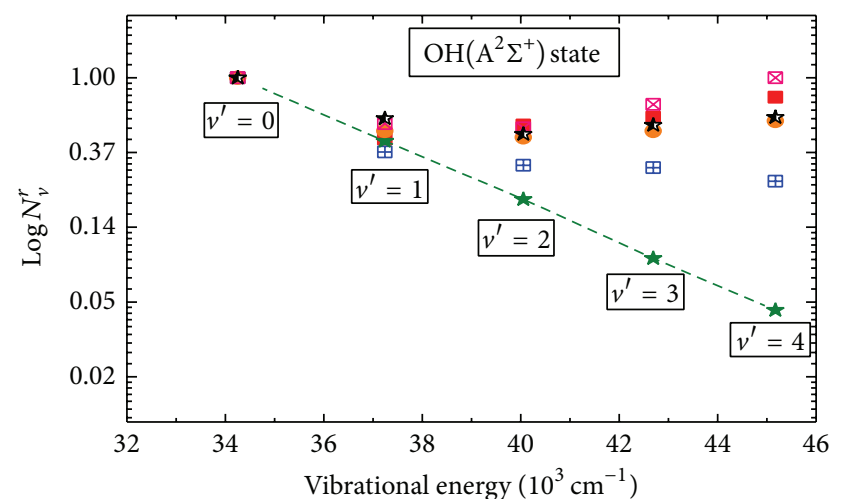

$\begin{array}{ll}\text { - } & 204 \mathrm{kHz} / 53 \mathrm{~W} \\ \boxplus & 20 \mathrm{kHz} / 30 \mathrm{~W} \\ \text { - } & 362 \mathrm{kHz} / 46 \mathrm{~W}\end{array}$

$\star \quad 609 \mathrm{kHz} / 46 \mathrm{~W}$

凶 $1057 \mathrm{kHz} / 54 \mathrm{~W}$

* - LIFBASE data:

$P=200$ bars $; T=5000 \mathrm{~K}$

FIGURE 2: Relative vibrational population distribution of the $\mathrm{OH}\left(\mathrm{A}^{2} \Sigma^{+}\right)$state as a function of vibrational energy for different ultrasonic frequencies. The green line demonstrates Boltzmann equilibrium distribution at $T=5000 \mathrm{~K}$ calculated using Lifbase database. Reproduced with permission from [22].

The electron temperature $\left(T_{e}\right)$ often significantly exceeds the vibrational $\left(T_{v}\right)$, rotational $\left(T_{r}\right)$, and translational $\left(T_{0}\right)$ temperatures: $T_{e}>T_{v}>T_{r} \approx T_{0}$ [39]. Table 2 summarizes the optimized values of $T_{e}$ and $T_{v}$ estimated from the simulated MBSL spectra [22]. It shows that the nonequilibrium plasma generated during the sonolysis of water sparged with argon obeys the classical inequality $T_{e}>T_{v}$. The $T_{e}$ value increases with the ultrasonic frequency from $\sim 8000 \mathrm{~K}$ $(\sim 0.7 \mathrm{eV})$ at $20 \mathrm{kHz}$ to $\sim 12000 \mathrm{~K}(\sim 1 \mathrm{eV})$ at $1057 \mathrm{kHz}$. These data clearly indicate that the acoustic collapse creates more drastic conditions at higher ultrasonic frequency. Another interesting phenomenon described in the literature is that the shape of $\mathrm{OH}^{*}$ radical SBSL spectrum obtained in water preequilibrated with $70 \mathrm{mbar}$ of Ar is very similar to that observed in MBSL spectra of argon-saturated water at $20 \mathrm{kHz}$, indicating the strong similarity of intrabubble conditions for SBSL and MBSL under certain experimental conditions [45].

The MBSL is strongly influenced by saturating noble gas. In xenon, the vibrational population distribution for $\mathrm{OH}^{\circ}$ radicals exhibits strong vibrational (Treanor) behavior even at low ultrasonic frequency. Moreover, the MBSL spectra of water exhibit much stronger $\mathrm{OH}\left(\mathrm{C}^{2} \Sigma^{+}-\mathrm{A}^{2} \Sigma^{+}\right)$emission bands in the presence of Xe compared to those in Ar [46]. In terms of nonequilibrium plasma model, the higher vibronic temperatures in Xe are the result of lower ionization potential of Xe compared to $\mathrm{Ar}$ (12.13 eV for Xe, $15.76 \mathrm{eV}$ for Ar). Lower ionization potential provides plasma with higher electron density or in other words with higher electron temperature.

The formation of nonequilibrium plasma inside the cavitation bubble is in strong correlation with the isotope effects in MBSL spectra of heavy and light water observed recently by Ndiaye et al. [46]. Despite a very small variation in physicochemical properties of $\mathrm{H}_{2} \mathrm{O}$ and $\mathrm{D}_{2} \mathrm{O}$, their MBSL
TABLE 2: Estimated vibronic temperatures of intrabubble plasma versus ultrasonic frequency in argon-saturated water at $10-12^{\circ} \mathrm{C}$ [22].

\begin{tabular}{lcc}
\hline$f, \mathrm{kHz}$ & $T_{e}, \mathrm{~K}$ & $T_{v}, \mathrm{~K}$ \\
\hline 20 & 8000 & 5000 \\
204 & 9500 & 7600 \\
362 & 10000 & 8450 \\
609 & 11000 & 9050 \\
1057 & 12000 & 9800 \\
\hline
\end{tabular}

spectra exhibit a striking difference whatever the ultrasonic frequency. The $\mathrm{OH} / \mathrm{OD}\left(\mathrm{A}^{2} \Sigma^{+}-\mathrm{X}^{2} \Pi_{i}\right)$ emission bands exhibit not only the isotope shift but also spectral profile modifications indicating differences in the populations of the $\mathrm{OH} / \mathrm{OD}\left(\mathrm{A}^{2} \Sigma^{+}\right)$vibrational levels. The strong emission from $\mathrm{OH}\left(\mathrm{C}^{2} \Sigma^{+}\right)$excited state observed in light water is dramatically reduced in heavy water. The spectroscopic analysis of $\mathrm{OH} / \mathrm{OD}\left(\mathrm{A}^{2} \Sigma^{+}-\mathrm{X}^{2} \Pi_{i}\right)$ transitions revealed overpopulation of both $\mathrm{OH}^{*}$ and $\mathrm{OD}^{\circ}$ vibrational levels compared to Boltzmann equilibrium distribution. Moreover, the isotope effect for relative $\mathrm{OD} / \mathrm{OH}\left(\mathrm{A}^{2} \Sigma^{+}\right)$vibrational populations $\left(\alpha=N_{v}^{\mathrm{OD}} / N_{v}^{\mathrm{OH}}\right)$ does not follow an exponential Boltzmann function. The trend followed by $\ln \alpha$ can be explained by formation of "hotter" nonequilibrium plasma in $\mathrm{D}_{2} \mathrm{O}$ than in $\mathrm{H}_{2} \mathrm{O}$. Finally, the nonequilibrium model can contribute to an understanding of SBSL in sulfuric acid. Actually, the electron temperature is an average energy of the electrons in plasma [39]. The electron energy distribution function (EEDF) in nonequilibrium plasma is quite different from the quasiequilibrium Boltzmann distribution. Usually, the EEDF is described by Maxwellian or by Druyvesteyn distributions [39]. In both cases, for $T_{e} \sim 1 \mathrm{eV}$ nonequilibrium plasma can contain the electrons with an energy of tens of $\mathrm{eV}$. This would explain the emission bands of highly energetic ionized and excited species in SBSL spectra of $\mathrm{H}_{2} \mathrm{SO}_{4}$. In water, these species most probably are quenched by $\mathrm{H}_{2} \mathrm{O}$ molecules.

\section{Activating Molecules, Ions, and Solids with Acoustic Cavitation}

Finding of nonequilibrium plasma formation during acoustic cavitation represents a paradigm shift for sonochemistry. Instead of simplistic adiabatic heating model, the sonochemical processes can be considered in terms of more sophisticated plasma chemical approach allowing explaining the new effects in sonochemistry as well as in sonoluminescence. One of such processes is a carbon isotope effect during sonochemical carbon monoxide disproportionation in water [49]. The prolonged sonication of water with $20 \mathrm{kHz}$ ultrasound in the presence of $20 \% \mathrm{CO} / \mathrm{Ar}$ gas mixture yields a tiny amount of solid carbon-containing product. It was found that the composition of this product has some similarity with hydrated poly(carbon suboxide) which is known to be formed during CO disproportionation in plasma [39]. Moreover, the product of sonolysis was enriched with the ${ }^{13} \mathrm{C}$ isotope $(\alpha=1.053-1.055)$. This effect is inconsistent with equilibrium isotope effects described by Bigeleisen-Mayer 
theory which predicts the enrichment of reaction products with light isotopes [50]. By contrast, vibrational excitation of $\mathrm{CO}$ molecules in nonequilibrium plasma leads to reverse isotope effect or in other words to the enrichment of reaction products with heavy isotopes [39]. The population of highly vibrational states of $\mathrm{CO}$ molecule occurs through an anharmonic vibration-to-vibration pumping mechanism, known as Treanor effect. It is noteworthy that the nonequilibrium vibrational excitation of $\mathrm{OH}^{*}$ radicals discussed above is related to the same phenomena. One can conclude that observed isotope effects clearly indicate that the nonequilibrium plasma inside the cavitation bubbles can influence not only the MBSL spectra but also the sonochemical reactions.

In principal, each cavitation bubble can be considered as a plasmochemical microreactor providing highly energetic processes at almost room temperature of the bulk solution. The photons and the "hot" particles produced inside the bubble enable exciting the nonvolatile species in solutions, thus increasing their chemical reactivity. For example, power ultrasound enables the excitation of lanthanide ions [51, 52] and uranyl $\mathrm{UO}_{2}{ }^{2+}$ ions [53] in aqueous acidic solutions. The mechanism of excitation involves two stages: sonophotoluminescence (excitation with photons emitted by collapsing bubble) dominates in diluted solutions, and collisional excitation with "hot" particles would add its contribution at higher metal ions concentration. It should be emphasized that the nonradiative deexcitation pathways play more important role in MBSL of Ln(III) and U(VI) ions compared to photoluminescence due to the quenching with sonolytical products $\left(\mathrm{H}_{2} \mathrm{O}_{2}, \mathrm{H}_{2}\right.$, etc.).

Oxidizing properties of power ultrasound in aqueous solutions related to the sonochemical production of $\mathrm{OH}^{*}$ radicals and hydrogen peroxide are known for a long time [8]. The sonochemical reduction processes are less common. Recently, the reduction of Pt(IV) under the action of $20 \mathrm{kHz}$ ultrasound in pure water has been reported [47]. In the presence of argon, reduction occurs by hydrogen issued from hemolytic water molecule split. However, Pt(IV) ion reduction appears to be slow at these conditions due to the formation of oxidizing species $\left(\mathrm{OH}^{\circ}, \mathrm{H}_{2} \mathrm{O}_{2}\right)$ leading to reoxidation of intermediate $\mathrm{Pt}(\mathrm{II})$ ions. Sonochemical reduction is accelerated manifold in the presence of formic acid or $\mathrm{CO} / \mathrm{Ar}$ gas mixture. Both $\mathrm{CO}$ and $\mathrm{HCOOH}$ act as $\mathrm{OH}^{*}$ radical scavengers and reducing agents. Sonolysis of $\mathrm{Pt}(\mathrm{IV})$ in aqueous solutions provides an innovative synthetic route to obtain monodispersed Pt nanoparticles without any templates or capping agents. The sonochemical process with $\mathrm{CO} / \mathrm{Ar}$ gas mixture in pure water yields $\mathrm{Pt}$ nanoparticles within the range of 2-3 nm highly stable towards sedimentation (Figure 3). It noteworthy that the high efficiency of the reduction process at low frequency ultrasonic irradiation is a further benefit for the controllable Pt nanoparticles deposition on various specific supports even on thermosensitive materials like polymer beads [54]. The sonochemical reduction of $\mathrm{Au}(\mathrm{III})$ ions to $\mathrm{Au}^{0}$ in argon-saturated water also has been observed by Caruso et al. [55].

The acoustic cavitation is accompanied not only by generation of chemically active species but also by strong

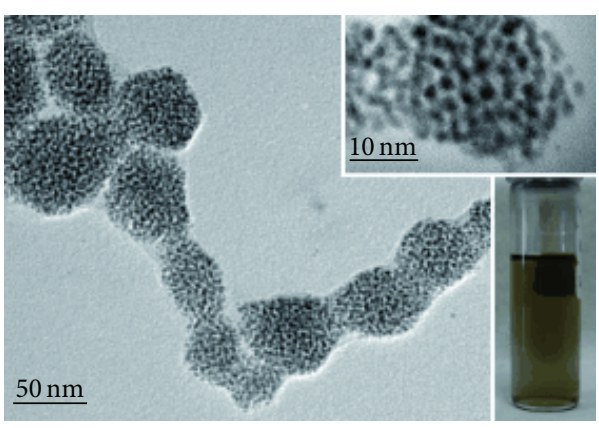

FIGURE 3: TEM image of Pt nanoparticles obtained under ultrasonic irradiation in pure water with $\mathrm{Ar} / \mathrm{CO}$ atmosphere. Picture of obtained brown colloid is given as an inset. Reproduced with permission from [47].

shear forces arising around violently imploding bubbles. Ultrasonic chain scission of polymers in solutions under the effect of these forces is known for a long time [56]. Recently the ultrasonic activation of homogeneous latent catalysts has been reported $[57,58]$. It was shown that the mechanochemical scission of a metal-ligand bond triggered with low-frequency ultrasound may be used to release the innate catalytic activity of either the ligand or the metal.

Actually, the mechanical forces generated by cavitation play much more important role in heterogeneous systems than in homogeneous solutions. The first observation of cavitation erosion has been reported by Thorneycroft and Barnaby [59] much earlier than any data on sonochemistry or sonoluminescence. They observed that the propeller of torpedo-boat destroyer became pitted and eroded over a relatively short operation period. This phenomenon is explained by asymmetric bubble collapse near interface which generates microjets and shock waves proving intense pressure and temperature gradients in the local vicinity. Today, the use of power ultrasound to enhance the reactivity of solids has become a routine technique in heterogeneous catalysis and various cleaning [4] and extraction [5] processes. In general, the mechanical effects of ultrasound are much stronger at low-frequency ultrasound compared to high ultrasonic frequency which is related to the decrease of bubble size with the increase of ultrasonic frequency. Recently it was found that the light emitted by an acoustically driven cloud of cavitation bubbles enables exciting $\mathrm{Tb}$ (III) contained in a (Ce,Tb) $\mathrm{PO}_{4}$ solid extended matrix [48]. The MBSL spectrum shown in Figure 4 demonstrates the $\mathrm{Tb}(\mathrm{III})$ light emission resulting from ${ }^{5} \mathrm{D}_{4}{ }^{-} \mathrm{F}_{j}$ f-f transitions along with $\mathrm{OH}(\mathrm{A}-\mathrm{X})$ and continuum emission typical for MBSL in water saturated with argon. Finally, the strong luminescence and even soft XRay emission (0.7-1.2 keV) have been recently reported during cavitation generated by high-pressure spindle oil jet at $P \geq$ 80-90 bar using narrow dielectric channels [60]. Detailed study of this phenomenon allowed concluding that the XRay emission originates from the excitation of the jet surface atoms by the cavitation-induced shock waves. Note here that the latter process is triggered by hydrodynamic cavitation. In 


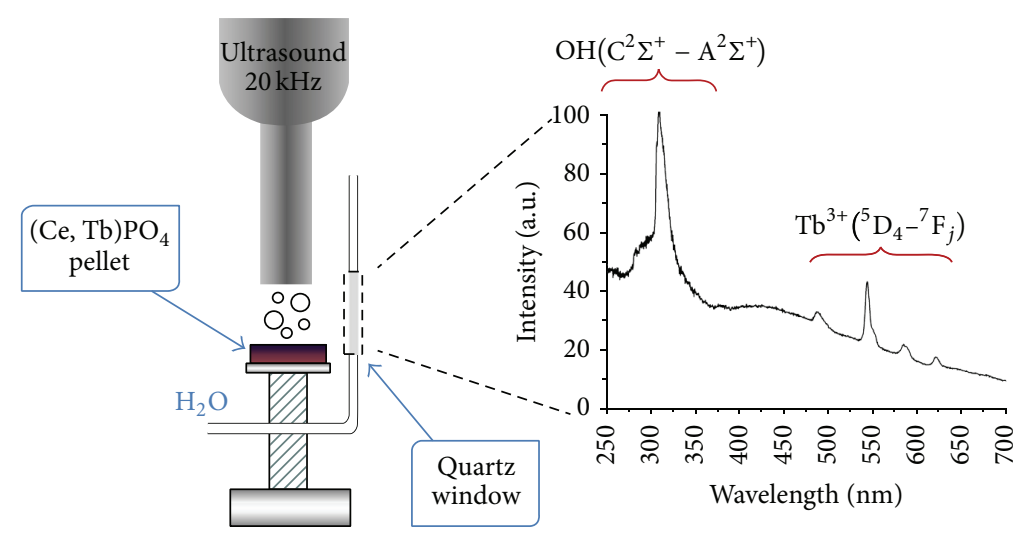

FIGURE 4: Simplified representation of the experimental setup and MBSL spectrum obtained for the sonication of $\left(\mathrm{Ce}_{0.9} \mathrm{~Tb}_{0.1}\right) \mathrm{PO}_{4}$ pellet with optimized horn-sample distance and focusing near the ultrasonic horn $\left(9^{\circ} \mathrm{C}, \mathrm{Ar}, f=20 \mathrm{kHz}, I=18 \mathrm{~W} \cdot \mathrm{cm}^{-2}\right)$. Reproduced with permission from [48].

contrast to the acoustic cavitation, hydrodynamic cavitation can be produced by passing a liquid through a constricted channel at a specific velocity or by mechanical rotation of an object through a liquid. The effects of hydrodynamic cavitation are generally recognized as similar to those of lowfrequency power ultrasound [4].

\section{Conclusions}

In summary, the recent spectroscopic investigations of sonoluminescence provide the strong evidence for nonequilibrium plasma formation during the acoustic collapse. The MBSL spectra of water saturated with noble gases reveal $\mathrm{OH}\left(\mathrm{A}^{2} \Sigma^{+}-\mathrm{X}^{2} \Pi_{i}\right)$ and $\mathrm{OH}\left(\mathrm{C}^{2} \Sigma^{+}-\mathrm{A}^{2} \Sigma^{+}\right)$emission bands and a broad continuum ranging from UV to NIR part of the emission spectra. The most probable mechanism of the $\mathrm{OH}\left(\mathrm{C}^{2} \Sigma^{+}\right)$ state production involves excitation of the water molecule by electron impact which is inconsistent with adiabatic heating model of cavitation dominated today in sonochemistry. The detailed analysis of $\mathrm{OH}\left(\mathrm{A}^{2} \Sigma^{+}-\mathrm{X}^{2} \Pi_{i}\right)$ emission bands revealed vibrational overpopulation of $\mathrm{OH}\left(\mathrm{A}^{2} \Sigma^{+}\right)$state. At low ultrasonic frequency, weakly excited plasma with Brau vibrational distribution is formed. By contrast, at high-frequency ultrasound, the plasma inside collapsing bubbles exhibits Treanor behavior typical for strong vibrational excitation. The vibronic temperatures $\left(T_{v}, T_{e}\right)$ increase with ultrasonic frequency indicating more drastic intrabubble conditions at high-frequency ultrasound. In xenon, the vibrational population distribution for $\mathrm{OH}^{\bullet}$ radicals exhibits Treanor behavior even at low ultrasonic frequency. Moreover, the MBSL spectra of water exhibit much stronger $\mathrm{OH}\left(\mathrm{C}^{2} \Sigma^{+}-\mathrm{A}^{2} \Sigma^{+}\right)$emission bands in the presence of Xe compared to those in Ar. In terms of nonequilibrium plasma model, the higher vibronic temperatures in $\mathrm{Xe}$ are the result of lower ionization potential of Xe compared to $\mathrm{Ar}$ (12.13 eV for Xe, $15.76 \mathrm{eV}$ for Ar). Lower ionization potential provides plasma with higher electron density or in other words with higher electron temperature.

The spectroscopic study of SBSL in sulfuric acid in the presence of noble gases revealed emission lines from ionized atoms $\mathrm{Xe}^{+}, \mathrm{Kr}^{+}$, and $\mathrm{Ar}^{+}$with the energies ranging from $26.0 \mathrm{eV}$ to $34.2 \mathrm{eV}$. However, the gas temperature obtained at the same conditions is only about $1 \mathrm{eV}$. Such a discrepancy cannot be understood by presuming adiabatic heating during bubble collapse, but it can be explained by the formation of nonequilibrium plasma with an electron temperature higher than the gas temperature. The future research should be focused on the understanding of physical process leading to the intrabubble plasma formation.

In principal, each cavitation bubble can be considered as a plasma chemical microreactor providing highly energetic processes at almost room temperature of the bulk solution. The photons and the "hot" particles produced inside the bubble enable the excitation of nonvolatile species in solutions, thus increasing their chemical reactivity. For example, the mechanism of ultrabright sonoluminescence of uranyl ions in acidic solutions is influenced by uranium concentration: photons absorption/reemission in diluted solutions and excitation via collisions with "hot" particles contributes at higher uranyl concentration. Chemical species produced by cavitation bubbles can be used for the synthesis of metallic nanoparticles without any templates or capping agents.

\section{Conflict of Interests}

The author declares that there is no conflict of interests regarding the publication of this paper.

\section{References}

[1] W. T. Richards and A. L. Loomis, "The chemical effects of high frequency sound waves I. A preliminary survey," Journal of the American Chemical Society, vol. 49, no. 12, pp. 3086-3100, 1927.

[2] F. O. Schmitt, C. H. Johnson, and A. R. Olson, "Oxidations promoted by ultrasonic radiation," Journal of the American Chemical Society, vol. 51, no. 2, pp. 370-375, 1929.

[3] E. A. Neppiras, "Acoustic cavitation," Physics Reports, vol. 61, no. 3, pp. 159-251, 1980.

[4] T. J. Mason and J. P. Lorimer, Applied Sonochemistry: The Uses of Power Ultrasound in Chemistry and Processing, Wiley-VCH, 
Weinheim, Germany, 2002.

[5] F. Chemat, Ed., Éco-Extraction du Végétal, Dunod, Paris, France, 2011.

[6] M. Ashokkumar, D. Sunartio, S. Kentish et al., "Modification of food ingredients by ultrasound to improve functionality: a preliminary study on a model system," Innovative Food Science \& Emerging Technologies, vol. 9, no. 2, pp. 155-160, 2008.

[7] H. Feng, G. V. Barbosa-Cánovas, and J. Weiss, Ultrasound Technologies for Food and Bioprocessing, Springer, New York, NY, USA, 2011.

[8] Y. G. Adewuyi, "Sonochemistry: environmental science and engineering applications," Industrial \& Engineering Chemistry Research, vol. 40, no. 22, pp. 4681-4715, 2001.

[9] N. N. Mahamuni and Y. G. Adewuyi, "Advanced oxidation processes (AOPs) involving ultrasound for waste water treatment: a review with emphasis on cost estimation," Ultrasonics Sonochemistry, vol. 17, no. 6, pp. 990-1003, 2010.

[10] J. H. B. Bang and K. S. Suslick, "Applications of ultrasound to the synthesis of nanostructured materials," Advanced Materials, vol. 22, no. 10, pp. 1039-1059, 2010.

[11] S. I. Nikitenko, L. Venault, R. Pflieger, T. Chave, I. Bisel, and P. Moisy, "Potential applications of sonochemistry in spent nuclear fuel reprocessing: a short review," Ultrasonics Sonochemistry, vol. 17, no. 6, pp. 1033-1040, 2010.

[12] N. Marinesco and J. J. Trillat, "Action des ultrasons sur les plaques photographiques," in Proceedings of the Royal Academy of Sciences, vol. 196, pp. 858-860, Amsterdam, The Netherlands, 1933.

[13] H. Frenzel and H. Schultes, "Luminescence in water carrying supersonic waves," Zeitschrift für Physikalische Chemie, vol. 27, pp. B421-B424, 1934.

[14] K. Yosioka and A. Omura, "The light emission from a single bubble driven by ultrasound and the spectra of acoustic oscillations," in Proceedings of the Annual Meetings of the Acoustical Society of Japan, pp. 125-126, Tokyo, Japan, 1962.

[15] P. R. Temple, Sonoluminescence from the gas in a single bubble [M.S. thesis], University of Vermont, Burlington, Vt, USA, 1970.

[16] D. F. Gaitan and L. A. Crum, "Sonoluminescence from single bubbles," The Journal of the Acoustical Society of America, vol. 87, supplement 1, p. S141, 1990.

[17] B. P. Barber and S. J. Putterman, "Observation of synchronous picosecond sonoluminescence," Nature, vol. 352, no. 6333, pp. 318-320, 1991.

[18] B. P. Barber, R. A. Hiller, R. Löfstedt, S. J. Putterman, and K. R. Weninger, "Defining the unknowns of sonoluminescence," Physics Reports, vol. 281, no. 2, pp. 65-143, 1997.

[19] S. J. Putterman and K. R. Weninger, "Sonoluminescence: how bubbles turn sound into light," Annual Review of Fluid Mechanics, vol. 32, pp. 445-476, 2000.

[20] W. Lauterborn, "Cavitation by laser light," Acustica, vol. 31, pp. 51-78, 1974

[21] F. R. Young, Sonoluminescence, CRC Press, Middlesex, UK, 2005.

[22] A. A. Ndiaye, R. Pflieger, B. Siboulet, J. Molina, J. F. Dufreche, and S. I. Nikitenko, "Nonequilibrium vibrational excitation of $\mathrm{OH}$ radicals generated during multibubble cavitation in water," The Journal of Physical Chemistry A, vol. 116, no. 20, pp. 48604867, 2012.

[23] M. S. Plesset, “The dynamics of cavitation bubbles," Journal of Applied Mechanics, vol. 16, pp. 277-282, 1949.
[24] M. S. Plesset and D. Y. Hsieh, "Theory of gas bubble dynamics in oscillating pressure fields," Physics of Fluids, vol. 3, no. 6, pp. 882-892, 1960.

[25] T. G. Leighton, The Acoustic Bubble, Academic Press, London, UK, 1994.

[26] W. C. Moss, "Understanding the periodic driving pressure in the Rayleigh-Plesset equation," The Journal of the Acoustical Society of America, vol. 101, no. 2, pp. 1187-1190, 1997.

[27] W. Lauterborn and T. Kurz, "Physics of bubble oscillations," Reports on Progress in Physics, vol. 73, no. 10, Article ID 106501, 2010.

[28] K. S. Suslick and D. J. Flannigan, "Inside a collapsing bubble: sonoluminescence and the conditions during cavitation," Annual Review of Physical Chemistry, vol. 59, pp. 659-683, 2008.

[29] M. Minnaert, "On musical air-bubbles and the sound of running water," Philosophical Magazine, vol. 16, no. 104, pp. 235$248,1933$.

[30] L. Rayleigh, "On the pressure developed in a liquid during the collapse of a spherical cavity," Philosophical Magazine, vol. 34, no. 200, pp. 94-98, 1917.

[31] B. E. Noltingk and E. A. Neppiras, "Cavitation produced by ultrasonics," Proceedings of the Physical Society B, vol. 63, no. 9, article 305, pp. 674-685, 1950.

[32] H. Flynn, "Cavitation dynamics. II. Free pulsations and models for cavitation bubbles," The Journal of the Acoustical Society of America, vol. 58, no. 6, pp. 1160-1170, 1975.

[33] M. A. Margulis, "On the mechanism of multibubble cavitation," Russian Journal of Physical Chemistry, vol. 80, pp. 1908-1913, 2006.

[34] B. Gompf, R. Gunther, G. Nick, R. Pecha, and W. Eisenmenger, "Resolving sonoluminescence pulse width with time-correlated single photon counting," Physical Review Letters, vol. 79, no. 7, pp. 1405-1408, 1997.

[35] R. A. Hiller, S. J. Putterman, and K. R. Weninger, "Timeresolved spectra of sonoluminescence," Physical Review Letters, vol. 80, no. 5, pp. 1090-1093, 1998.

[36] M. J. Moran and D. Sweider, "Measurements of sonoluminescence temporal pulse shape," Physical Review Letters, vol. 80, no. 22, pp. 4987-4990, 1998.

[37] M. Brenner, S. Hilgenfeldt, and D. Lohse, "Single-bubble sonoluminescence," Reviews of Modern Physics, vol. 74, no. 2, pp. 425-483, 2002.

[38] D. J. Flannigan and K. S. Suslick, "Plasma formation and temperature measurement during single-bubble cavitation," Nature, vol. 434, no. 7029, pp. 52-55, 2005.

[39] A. Fridman, Plasma Chemistry, Cambridge University Press, Cambridge, Mass, USA, 2008.

[40] D. J. Flannigan and K. S. Suslick, "Plasma line emission during single-bubble cavitation," Physical Review Letters, vol. 95, no. 4, Article ID 044301, 2005.

[41] W. Chen, W. Huang, Y. Liang, X. Gao, and W. Cui, "Timeresolved spectra of single-bubble sonoluminescence in sulfuric acid with a streak camera," Physical Review E: Statistical, Nonlinear, and Soft Matter Physics, vol. 78, no. 3, part 2, Article ID 035301, 2008.

[42] E. B. Flint and K. S. Suslick, "The temperature of cavitation," Science, vol. 253, no. 5026, pp. 1397-1399, 1991.

[43] N. C. Eddingsaas and K. S. Suslick, "Evidence for a plasma core during multibubble sonoluminescence in sulfuric acid," Journal of the American Chemical Society, vol.129, no. 13, pp. 3838-3839, 2007. 
[44] R. Pflieger, H. P. Brau, and S. I. Nikitenko, "Sonoluminescence from $\mathrm{OH}\left(\mathrm{C}^{2} \Sigma^{+}\right)$and $\mathrm{OH}\left(\mathrm{A}^{2} \Sigma^{+}\right)$radicals in water: evidence for plasma formation during multibubble cavitation," Chemistry: A European Journal, vol. 16, no. 39, pp. 11801-11803, 2010.

[45] J. Schneider, R. Pflieger, S. I. Nikitenko, D. Shchukin, and H. Möhwald, "Line emission of sodium and hydroxyl radicals in single-bubble sonoluminescence," The Journal of Physical Chemistry A, vol. 115, no. 2, pp. 136-140, 2011.

[46] A. A. Ndiaye, R. Pflieger, B. Siboulet, and S. I. Nikitenko, "The origin of isotope effects in sonoluminescence spectra of heavy and light water," Angewandte Chemie, vol. 52, no. 9, pp. 24782481, 2013.

[47] T. Chave, N. M. Navarro, S. Nitsche, and S. I. Nikitenko, "Mechanism of $\mathrm{Pt}^{\mathrm{IV}}$ sonochemical reduction in formic acid media and pure water," Chemistry: A European Journal, vol. 18, no. 13, pp. 3879-3885, 2012.

[48] M. Virot, R. Pflieger, J. Ravaux, and S. I. Nikitenko, "Sonoluminescence of $\mathrm{Tb}(\mathrm{III})$ at the extended solid-liquid interface," The Journal of Physical Chemistry C, vol. 115, no. 21, pp. 10752-10756, 2011.

[49] S. I. Nikitenko, P. Martinez, T. Chave, and I. Billy, "Sonochemical disproportionation of carbon monoxide in water: evidence for Treanor effect during multibubble cavitation," Angewandte Chemie, vol. 48, no. 50, pp. 9529-9532, 2009.

[50] J. Blgeleisen and M. G. Mayer, "Calculation of equilibrium constants for isotopic exchange reactions," The Journal of Chemical Physics, vol. 15, no. 5, pp. 261-267, 1947.

[51] G. L. Sharipov, R. K. Gainetdinov, and A. M. Abdrakhmanov, "Sonoluminescence of aqueous solutions of lanthanide salts," Russian Chemical Bulletin, vol. 52, no. 9, pp. 1969-1973, 2003.

[52] R. Pflieger, J. Schneider, B. Siboulet, H. Möhwald, and S. I. Nikitenko, "Luminescence of trivalent lanthanide ions excited by single-bubble and multibubble cavitations," The Journal of Physical Chemistry B, vol. 117, no. 10, pp. 2979-2984, 2013.

[53] R. Pflieger, V. Cousin, N. Barré, P. Moisy, and S. I. Nikitenko, "Sonoluminescence of uranyl ions in aqueous solutions," Chemistry: A European Journal, vol. 18, no. 2, pp. 410-414, 2012.

[54] T. Chave, A. Grunenwald, A. Ayral, P. Lacroix-Desmazes, and S. I. Nikitenko, "Sonochemical deposition of platinum nanoparticles on polymer beads and their transfer on the pore surface of silica matrix," Journal of Colloid and Interface Science, vol. 395, pp. 81-84, 2013.

[55] R. A. Caruso, M. Ashokkumar, and F. Grieser, "Sonochemical formation of gold sols," Langmuir, vol. 18, no. 21, pp. 7831-7836, 2002.

[56] A. M. Basedow and K. H. Ebert, "Ultrasonic degradation of polymer in solution," Advances in Polymer Science, vol. 22, pp. 83-148, 1977.

[57] A. Piermattei, S. Karthikeyan, and R. P. Sijbesma, "Activating catalysts with mechanical force," Nature Chemistry, vol. 1, no. 2, pp. 133-137, 2009.

[58] R. T. M. Jakobs and R. P. Sijbesma, "Mechanical activation of a latent olefin metathesis catalyst and persistence of its active species in ROMP," Organometallics, vol. 31, no. 6, pp. 2476-2481, 2012.

[59] J. Thorneycroft and S. W. Barnaby, "Torpedo-boat destroyers," Proceedings of the Institution of Civil Engineers, vol. 122, no. 1895, pp. 51-55, 1895.

[60] A. A. Kornilova, V. I. Vysotskii, N. N. Sysoev, and A. V. Desyatov, "Generation of X-rays at bubble cavitation in a fast liquid jet in dielectric channels," Journal of Surface Investigation, vol. 3, no. 2, pp. 275-283, 2009. 

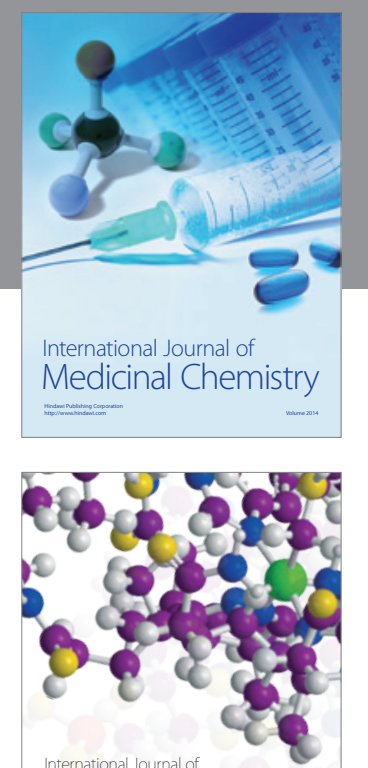

\section{Carbohydrate} Chemistry

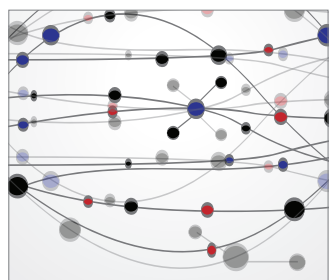

The Scientific World Journal
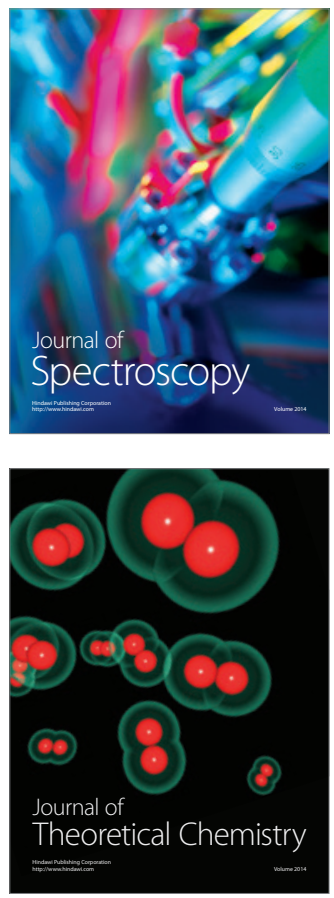
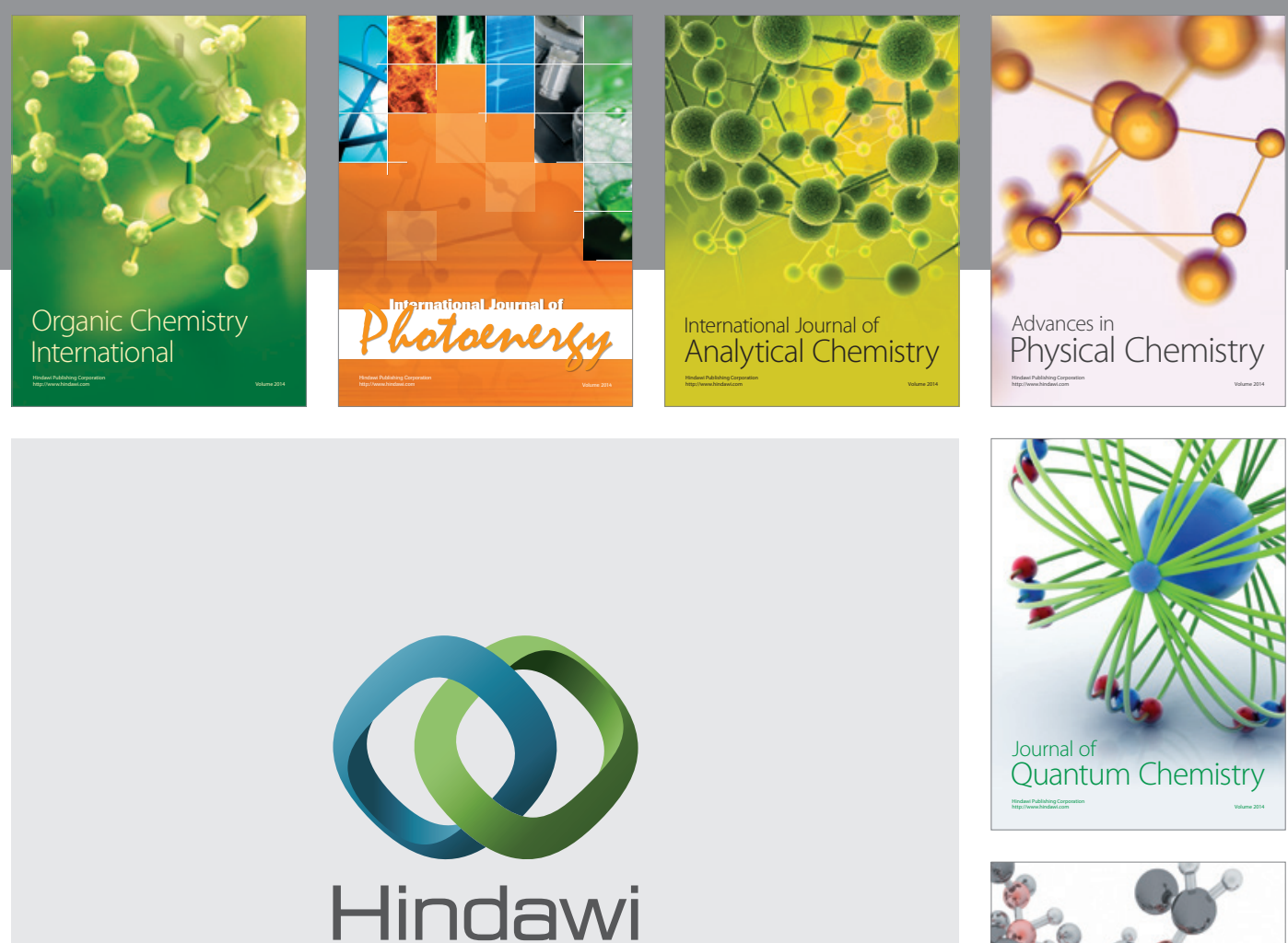

Submit your manuscripts at

http://www.hindawi.com

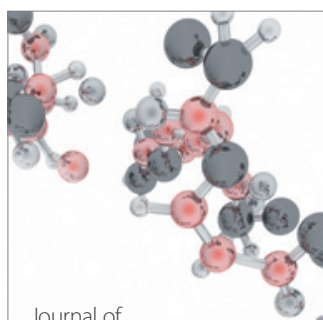

Analytical Methods

in Chemistry

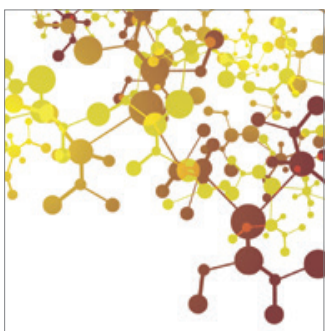

Journal of

Applied Chemistry

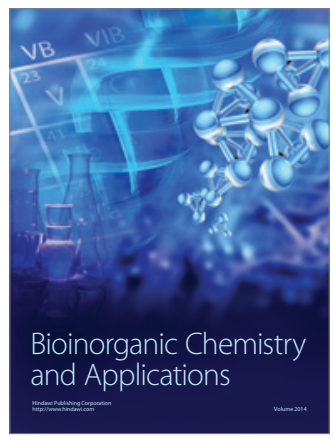

Inorganic Chemistry
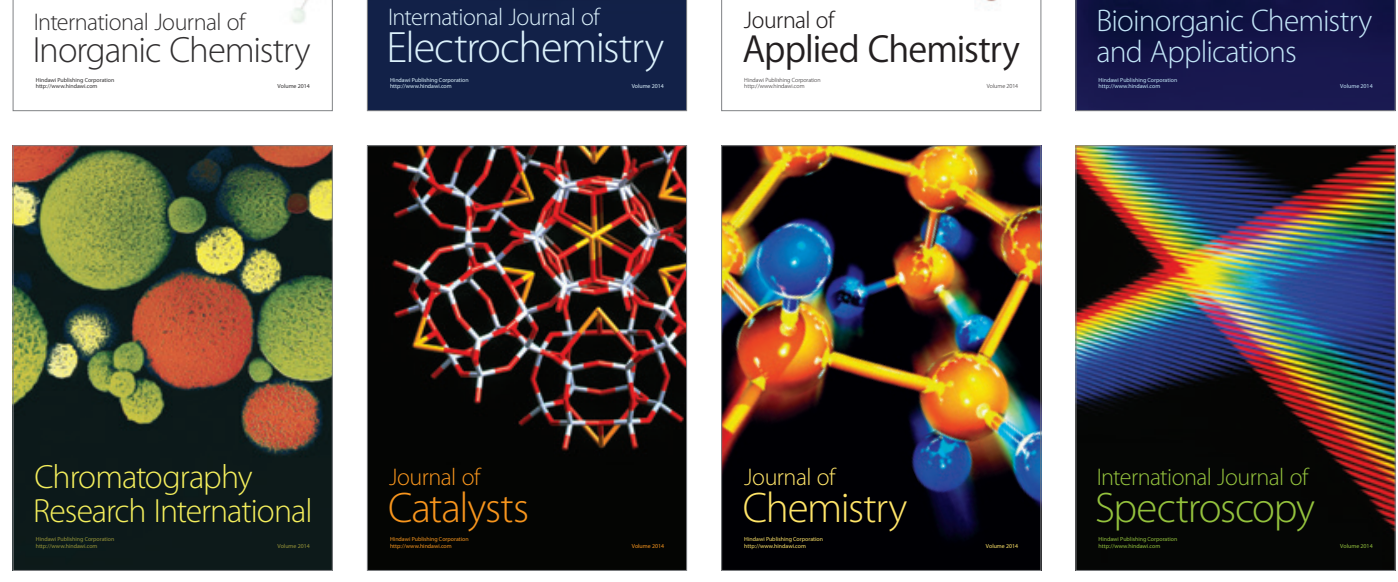